\title{
Effets de la valorisation des résidus de récolte sur la nutrition minérale du cotonnier et les rendements d'une rotation coton-maïs-sorgho dans l'Ouest du Burkina Faso
}

\author{
B. KOULIBALY ${ }^{1 *}$, O. TRAORE ${ }^{2}$, D. DAKUO ${ }^{3}$, N.P. ZOMBRE ${ }^{4}$ et D. BONDE ${ }^{5}$ \\ ${ }^{1}$ Institut de l'Environnement et de Recherches Agricoles (INERA), Programme coton 01 BP 208 Bobo- \\ Dioulasso 01, Burkina Faso. Tél : (226) 209721 05/70 2390 05, Fax : (226) 209701 59; E-mail: \\ bazoumana@hotmail.com \\ ${ }^{2}$ Programme Coton de l'Institut de l'Environnement et de Recherches Agricoles (INERA), 01 BP 208 Bobo- \\ Dioulasso 01, Burkina Faso.E-mail: ouola.traore@coraf.org \\ ${ }^{3}$ Développement de la Production Cotonnière de la Société Burkinabé des Fibres Textiles (SOFITEX). BP 147, \\ Bobo-Dioulasso, Burkina Faso. E-mail: ddakuo@yahoo.fr \\ ${ }^{4}$ Unité de Formation des Sciences de la Vie et de la Terre (UFR/SVT), Laboratoire de Biologie et Ecologie \\ Végétale, Université de Ouagadougou, E-mail : prosper.zombre@univ-ouaga.bf \\ ${ }^{5}$ Université Polytechnique de Bobo-Dioulasso (UPB), Institut du Développement Rural (IDR), Département \\ d'Agronomie. E-mail : desire06bonde@yahoo.fr. \\ *Auteur correspondant, Tél : (226) 209721 05/70 2390 05, E-mail: bazoumana@hotmail.com
}

\section{RESUME}

Une étude a été réalisée de 1982 à 2006 pour analyser les effets de la gestion des résidus de récolte sur la nutrition minérale des cotonniers et les rendements d'une rotation coton-maïs-sorgho. Dans un dispositif expérimental en blocs simples non randomisés, la gestion extensive où les résidus sont exportés (T1) est comparée à la gestion semi-intensive (T2) et à la gestion intensive des résidus de récolte (T3) recyclés en compost et fumier, respectivement. La nutrition minérale des cotonniers et les rendements des cultures ont été évalués. La nutrition des cotonniers en $\mathrm{N}, \mathrm{P}, \mathrm{K}$ et $\mathrm{S}$ est satisfaite et n'est pas améliorée par le compost ou le fumier, issu du recyclage des résidus de récolte. On observe au bout de 13 années, de fortes carences en azote $(\mathrm{F}(\mathrm{N})<69)$ et en potassium $(\mathrm{F}(\mathrm{K})<80)$ ainsi qu'une déficience en soufre uniquement sur les cotonniers recevant la fumure minérale. La durée d'exploitation des terres entraîne des baisses de rendement élevées avec l'exportation résidus de récolte (T1) sur le coton, le maïs et le sorgho. Le recyclage des résidus en compost ou fumier permet de réduire deux à trois fois ces baisses de rendement en 25 ans. L'étude recommande une gestion intégrée des résidus de récolte et l'utilisation du phosphate naturel pour la durabilité des systèmes de culture.

() 2010 International Formulae Group. All rights reserved.

Mots clés: résidus de récolte, compost, fumier, nutrition minérale, rendements.

\section{INTRODUCTION}

Les systèmes de cultures actuels au Burkina Faso contribuent à la dégradation des ressources naturelles notamment la fertilité des sols qui ne cesse de baisser au fil des années (Traoré et al., 2007). La mise en culture des sols en zone cotonnière, entraîne une acidification accentuée par l'utilisation quasi exclusive des engrais minéraux (Ouattara et al., 2006; Agoumé et Birang, 
2009). Le cotonnier est pourtant très sensible à cette dégradation de la fertilité des sols. L'apparition généralisée des symptômes de déficiences minérales entraîne d'importantes baisses de rendement et de la qualité du coton (Girma et al., 2007). La durabilité des systèmes de culture exige une gestion rationnelle de la fertilité des sols qui est loin d'être assurée à cause de l'insuffisance des restitutions organiques (Ouédraogo et al., 2006). Ainsi, dans les systèmes de production à base de coton et de céréales, les résidus de récolte sont brûlés ou pâturés, ce qui entraîne souvent des bilans minéraux déficitaires (Olina Bassala et al., 2008). Le maintien de la fertilité est compromis par cette mauvaise gestion des résidus de récolte et par le sousdosage des engrais minéraux importés à cause de leur coût élevé.

La nutrition minérale du cotonnier, notamment en azote, en phosphore et en potassium, constitue un facteur déterminant de la production et de la qualité de la fibre (Crozier et al., 2004 ; Reiter et al., 2008). Il est nécessaire de bien gérer la fertilité des sols et d'ajuster la fertilisation aux besoins des plantes (Vanlauwe et al., 2005). Le diagnostic foliaire est un outil précieux et adapté au suivi de la nutrition du cotonnier, permettant de déterminer le niveau de déficience minérale $\mathrm{au}$ moment du prélèvement foliaire (Olina Bassala et al., 2008). La satisfaction des besoins minéraux des cotonniers doit être privilégiée en vue de la rentabilité économique et de la durabilité des systèmes de culture coton-céréales.

Cette étude qui sert de référentiel technique, s'inscrit dans la dynamique de l'intensification et de la durabilité des systèmes de production en zone cotonnière. Le présent article a pour principal objectif d'analyser les effets de trois modes de gestion des résidus de récolte combinés aux engrais minéraux et au phosphate naturel sur l'alimentation minérale des cotonniers et sur les productions des cultures dans une rotation triennale coton-maïs-sorgho. L'évolution des caractéristiques chimiques du sol et les bilans culturaux dans ce système de culture ont été abordés dans un précédent article (Koulibaly et al., 2010).

\section{MATERIEL ET METHODES Site d'étude}

L'étude est conduite sur la ferme expérimentale de Boni $\left(3^{\circ} 26\right.$ ' de longitude Ouest, $11^{\circ} 32$ ' de latitude Nord et $302 \mathrm{~m}$ d'altitude) sur un sol ferrugineux tropical gravillonnaire. Le climat est du type sudsoudanien avec une saison pluvieuse allant de mai à octobre et une saison sèche de novembre à avril (Barro et al., 2009). La pluviométrie varie entre 723 et $1353 \mathrm{~mm}$ répartie sur 40 à 75 jours de pluie (Tableau 1). Cette pluviométrie, souvent mal répartie, est très irrégulière avec des années excédentaires (> $1100 \mathrm{~mm})$ et des années très déficitaires (< $800 \mathrm{~mm}$ ). Les déficits pluviométriques occasionnent des retards de semis et peuvent fortement compromettre les rendements des cultures.

\section{Dispositif expérimental}

Dans un dispositif en blocs simples non randomisés, six hectares sont cultivés en rotation coton-maïs-sorgho à raison de deux hectares par culture et par année. Trois modes de gestion des résidus de récoltes combinés à des apports de phosphate naturel $\left(25 \% \mathrm{P}_{2} \mathrm{O}_{5}\right.$ et $35 \% \mathrm{CaO}$ ) et d'engrais minéraux sont comparés.

T1 - Gestion extensive des résidus de récolte. Les tiges de maïs et de sorgho sont pâturées ou exportées de la parcelle. Tous les trois ans sur le maïs, $300 \mathrm{~kg} \mathrm{ha}^{-1}$ de phosphate naturel sont apportés au hersage. Ce traitement correspond à la pratique la plus proche de celle des paysans, considérée dans cette étude comme la gestion extensive des résidus de récolte.

T2 - Gestion semi-intensive des résidus de récolte. Les tiges de maïs sont enfouies au sol par un labour de fin d'hivernage tandis que les tiges de sorgho ( $4 \mathrm{t} \mathrm{ha}^{-1}$ en moyenne), sont 
recyclées en compost après 45 jours de broyage par 20 bœufs dans un parc où on apporte $300 \mathrm{~kg}$ de phosphate naturel. Les tiges broyées sont ensuite retirées du parc puis soumises à des arrosages et retournements au cours d'un compostage en tas qui dure 60 jours. Le compost obtenu est appliqué tous les trois ans sur le maïs à raison de $6 \mathrm{t} \mathrm{ha}^{-1}$ et contient en moyenne $28 \%$ de matière organique ; $2,2 \% \mathrm{~N} ; 1,9 \% \mathrm{P}_{2} \mathrm{O}_{5} ; 1,8 \% \mathrm{~K}_{2} \mathrm{O}$ et $0,3 \% \mathrm{SO}_{4}$.

T3 - Gestion intensive des résidus de récolte. Les tiges de maïs sont enfouies au sol par un labour de fin d'hivernage. Les tiges de sorgho $\left(4 \mathrm{t} \mathrm{ha}^{-1}\right)$ sont recyclées en fumier par la technique du parc d'hivernage (Berger, 1996). Après 60 jours de broyage par un troupeau de 20 bœufs, les tiges séjournent dans le parc durant l'hivernage et se décomposent uniquement sous l'action des eaux de pluie. Cela permet d'obtenir 18 mois après la récolte du sorgho, du fumier de parc qui contient $28 \%$ de matière organique ; $2,2 \% \mathrm{~N} ; 1,1 \% \mathrm{P}_{2} \mathrm{O}_{5}$; $1,7 \% \mathrm{~K}_{2} \mathrm{O}$ et $0,3 \% \mathrm{SO}_{4}$. Il est utilisé à la dose de $6 \mathrm{t} \mathrm{ha}^{-1}$ tous les trois ans sur le maïs avec $300 \mathrm{~kg} \mathrm{ha}^{-1}$ de phosphate naturel.

Dans les trois systèmes de gestion des résidus, les tiges de cotonnier sont coupées et brûlées en petits tas et leurs cendres sont enfouies au sol par le labour. La fertilisation minérale (engrais solubles et phosphate naturel) ramenée à l'année, apporte par hectare $46 \mathrm{~N}, 25 \mathrm{P}_{2} \mathrm{O}_{5}, 48 \mathrm{~K}_{2} \mathrm{O}, 18 \mathrm{SO}_{4}$ et $1 \mathrm{~B}_{2} \mathrm{O}_{3}$ sur le cotonnier, $74 \mathrm{~N}, 25 \mathrm{P}_{2} \mathrm{O}_{5}, 60 \mathrm{~K}_{2} \mathrm{O}$ sur le maïs, $46 \mathrm{~N}$ et $25 \mathrm{P}_{2} \mathrm{O}_{5}$ sur le sorgho.

\section{Matériel végétal}

Les variétés de cotonnier (MK 73, L.299.10.75, ISA 205 G, STAM 42, F 135, STAM 59 A, FK 290 et FK 37), de maïs (IRAT 171, SR 22 et SR 21) et de sorgho (Gnoffing et Sariasso 01) ont été utilisées. Les potentiels de rendement de ces variétés sont de 3 à $4 \mathrm{t} \mathrm{ha}^{-1}$ pour le cotonnier, de 4 à $5 \mathrm{t} \mathrm{ha}^{-1}$ pour le maïs et de 2 à $3 \mathrm{tha}^{-1}$ pour le sorgho.

\section{Conduite des opérations culturales}

Un labour du sol au tracteur à partir de mai précède les semis effectués du 20 mai au 10 juillet. Un démariage est pratiqué 15 jours après la levée afin de ramener les trois cultures à une densité théorique de 62500 plants $\mathrm{ha}^{-1}$. La lutte contre les mauvaises herbes est réalisée par des désherbages chimiques, manuels et mécaniques. Le programme de protection phytosanitaire vulgarisé en culture cotonnière a été suivi en appliquant le profénofos $\left(500 \mathrm{~g} \mathrm{ha}^{-1}\right), 30$ et 44 jours après la levée des cotonniers, l'association «lamdacyalotrhine (12 $\left.\mathrm{g} \mathrm{ha}^{-1}\right)$ profénofos $\left(200 \mathrm{~g} \mathrm{ha}^{-1}\right) »$ aux $58^{\grave{e}}$ et $72^{\grave{e}}$ jours puis l'association « cyperméthrine $\left(36 \mathrm{~g} \mathrm{ha}^{-1}\right)$ acétamipride $\left(8 \mathrm{~g} \mathrm{ha}^{-1}\right) »$ aux $86^{\mathrm{e}}$ et $100^{\text {è }}$ jours.

\section{Prélèvements foliaires et analyses de laboratoire}

Des prélèvements foliaires sont effectués chaque année sur les cotonniers au $70{ }^{\mathrm{e}}$ jour après le semis selon la méthode du diagnostic foliaire de l'IRCT (Braud, 1987). Les feuilles prélevées sont celles situées à l'aisselle d'une fleur ouverte se trouvant sur le premier nœud d'une branche fructifère le jour du prélèvement. Les teneurs en anions $(\mathrm{N}, \mathrm{P}, \mathrm{S}$, $\mathrm{Cl})$ sont déterminées sur les limbes et celles en cations $(\mathrm{K}, \mathrm{Ca}, \mathrm{Mg}$ et $\mathrm{Na}$ ) sur les pétioles. Durant 17 années (1982 à 1998), les fonctions de production en $\mathrm{N}, \mathrm{P}, \mathrm{K}$ et $\mathrm{S}$ ont été calculées à partir des formules de type $\mathrm{F}(\mathrm{X})=\mathrm{f}(\mathrm{N}, \mathrm{P}, \mathrm{K}$, S, p, F), proposées par Braud (1974) dans lesquelles $\mathrm{F}(\mathrm{X})$ est le rendement que donne une fumure complète dont on a soustrait l'élément $\mathrm{X}$. N, P, K et S exprimés en \%, représentent les teneurs de ces éléments dans la matière sèche, $p$ exprimé en gramme, est le poids sec de 30 feuilles séchées à $70{ }^{\circ} \mathrm{C}$ à l'étuve et où $\mathrm{F}$ est le niveau de floraison des plants au $70^{\grave{~}}$ jour après les semis. La nutrition est alors estimée optimale pour un élément, si la fonction de production est supérieure à 100 , bonne si elle est comprise entre 90 et 100, déficiente si elle 
est comprise entre 80 et 90 et carencée si la fonction de production est inférieure à 80 .

Les teneurs en éléments minéraux des cotonniers ont été déterminées par des analyses effectuées au laboratoire du Bureau National des Sols (BUNASOLS) du Burkina Faso selon les méthodes qui y sont utilisées (Bunasols, 1987).

\section{Evaluation des rendements}

Les récoltes du coton, du maïs et du sorgho sont réalisées par traitement afin de déterminer les rendements des trois cultures chaque année.

\section{Analyses statistiques}

Les données collectées ont été soumises à une analyse de variance réalisée avec le logiciel XLSTAT 6.1.9. Le test de Fisher a été utilisé pour la séparation des moyennes lorsque l'analyse de variance révèle des différences significatives entre les traitements au seuil de signification de $5 \%$.

\section{RESULTATS}

Effets de la gestion des résidus de récolte sur la nutrition minérale des cotonniers

Durant les 10 premières années, la nutrition des cotonniers en $\mathrm{N}, \mathrm{P}, \mathrm{K}$ et $\mathrm{S}$ a été satisfaite par la fumure minérale utilisée en gestion extensive des résidus de récolte (T1) et n'a pas été améliorée par les apports additionnels de compost (T2) ou de fumier (T3) (Figure 1). L'évolution de la nutrition des cotonniers en azote a été marquée par trois phases: une nutrition optimale $(\mathrm{F}(\mathrm{N})>100) \mathrm{au}$ cours des quatre premières années (1982 à 1985), puis une bonne nutrition de 1986 à $1993(90<\mathrm{F}(\mathrm{N})<100)$, suivie d'une nutrition carencée de 1994 à $1996(41<\mathrm{F}(\mathrm{N})<69)$ (Figure 1a). Durant cette période de carence, on a observé avec une pluviosité de 1006 à 1209 mm (Tableau 1), que les teneurs en azote des cotonniers ont chuté à des valeurs comprises entre 1,96 et $2,03 \%$ sur tous les traitements (Tableau 2). La nutrition en phosphore des cotonniers a été bonne pendant les 10 premières années $(\mathrm{F}(\mathrm{P})>90)$ et s'est même améliorée de 1991 à 1998, période durant laquelle une nutrition optimale a été observée sur tous les traitements $(\mathrm{F}(\mathrm{P})>100)$ (Figure 1b). La nutrition des cotonniers en potassium a été bonne $(90<\mathrm{F}(\mathrm{K})<100)$ et relativement stable pendant plus de 12 années sans être influencée par les traitements. Une carence potassique a été observée sur les cotonniers en 1994, après 13 années de culture continue $(\mathrm{F}(\mathrm{K})<80)$ (Figure 1c). Les teneurs des cotonniers en potassium, fortement dépendantes de la pluviosité, sont significativement différentes $(\mathrm{p}<0,01)$ pour les cycles de rotation (Tableau 2). La nutrition des cotonniers en soufre a été relativement stable : bonne pendant huit années jusqu'en $1989(90<\mathrm{F}(\mathrm{S})<100)$, puis optimale à partir de 1990. Après 15 années, une déficience en soufre est apparue de façon brutale $(80<\mathrm{F}$ (S) $<90$ ), uniquement sur les cotonniers témoins recevant la fumure minérale seule en gestion extensive des résidus de récolte (Figure 1d). Les teneurs en soufre comprises entre 0,37 et $1 \%$ indiquent dans l'ensemble une nutrition correcte des cotonniers (Tableau 2). En 17 années d'observation, l'exportation des résidus de récolte (T1) ou leur recyclage (T2 et T3) n'ont pas montré d'effets significatifs sur les teneurs en calcium $(1,15$ à $2,52 \%)$, en magnésium $(0,31$ à $0,66 \%)$ et en sodium $(0,04$ à $0,52 \%)$. De même, les teneurs en chlore de 0,73 à $1,98 \%$ et en bore de 30,96 à 39,33 mg $\mathrm{kg}^{-1}$ reflètent dans l'ensemble une nutrition minérale correcte des cotonniers.

\section{Evolution du rendement des cultures}

Les modes de gestion des résidus de récolte n'ont pas eu d'effets significatifs sur les rendements du coton et du maïs pendant 12 ans de culture, ni sur ceux du sorgho pendant 15 ans (Figures 2, 3 et 4). En 25 années de culture continue, les restitutions au sol du compost (T2) ou du fumier (T3) ont toujours permis d'obtenir des rendements plus élevés que ceux du témoin sans aucune restitution organique (T1). On observe avec la durée de mise en culture des terres, une baisse 
des rendements quelle que soit la gestion des résidus de récolte. Cette baisse des rendements est accentuée par l'exportation des résidus de récolte. Pour le coton, l'évolution des rendements en gestion extensive des résidus peut alors s'exprimer suivant l'équation : Y $\left(\mathrm{kg} \mathrm{ha}^{-1}\right)=-22,12 \mathrm{X}+1778$, avec $\mathrm{r}^{2}=0,98$ et où $\mathrm{X}$ est le nombre d'années de mise en culture des terres (Figure 2). La baisse de rendement après 25 années d'apport de fumure minérale exclusive sur le coton était 2,7 fois plus importante que celle observée avec l'apport combiné du compost et de la fumure minérale.

Dans le cas du maïs (Figure 3) et du sorgho (Figure 4), les apports de compost (T2) ou de fumier (T3), sans empêcher la baisse des rendements, l'ont réduit deux à trois fois par rapport à la fumure minérale seule (T1) en 25 ans d'exploitation des terres.

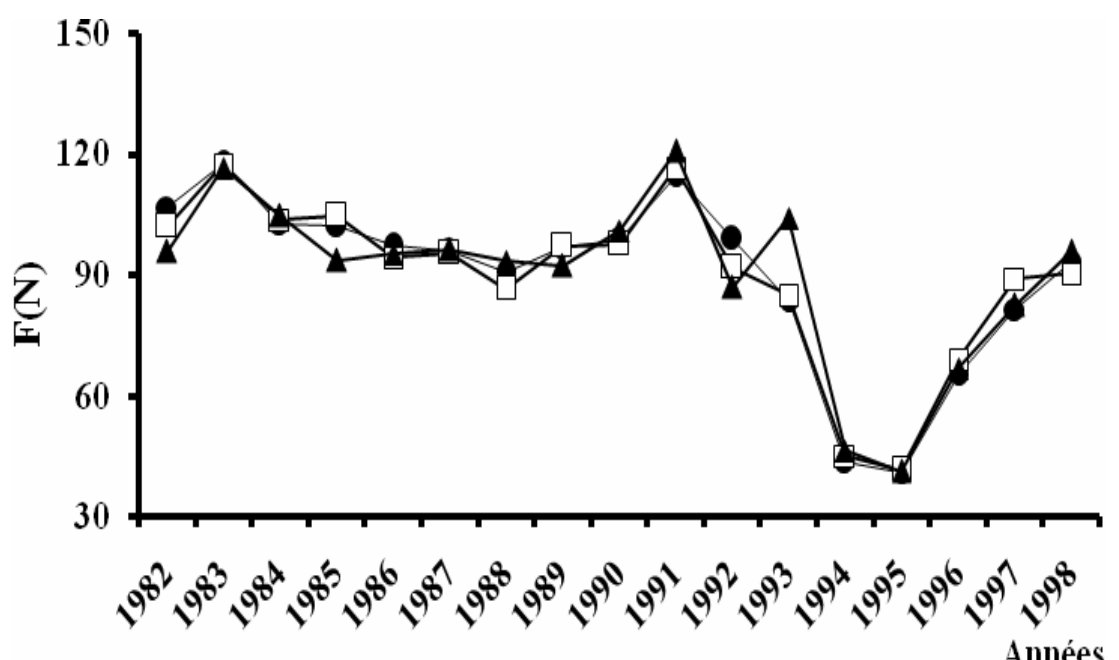

a) Nutrition en azote

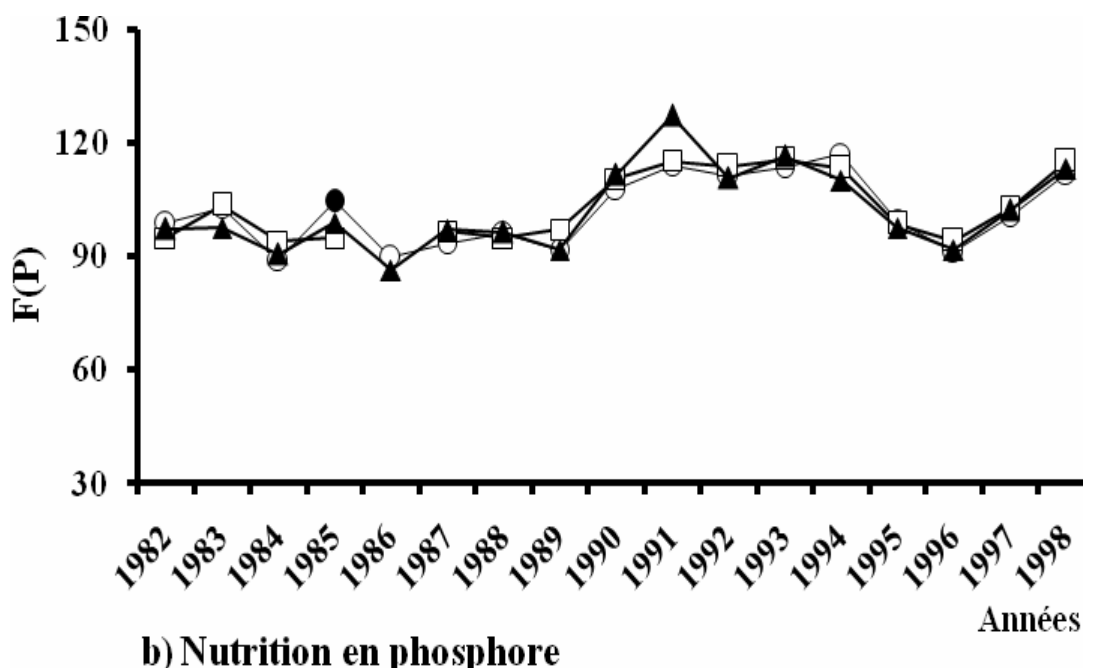




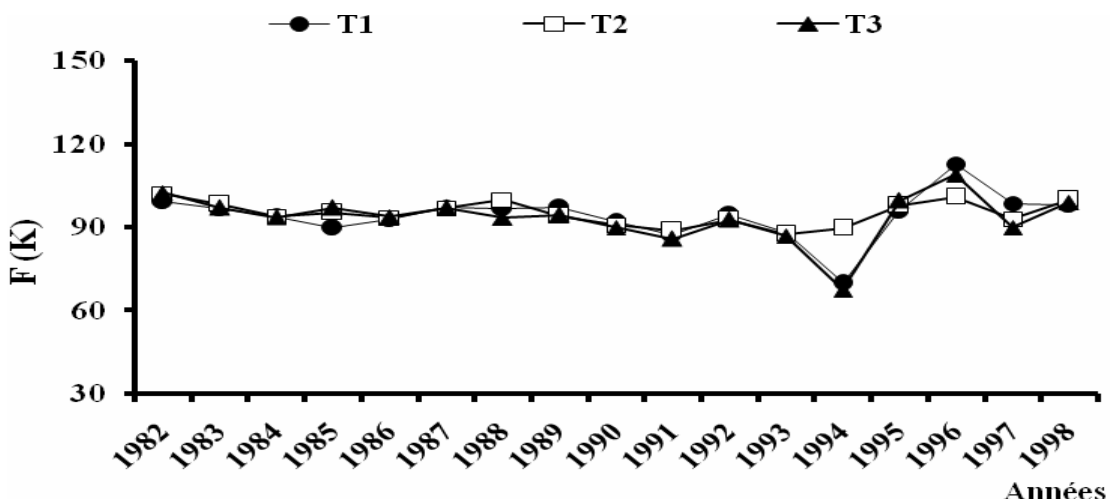

c) Nutrition en pota ssium

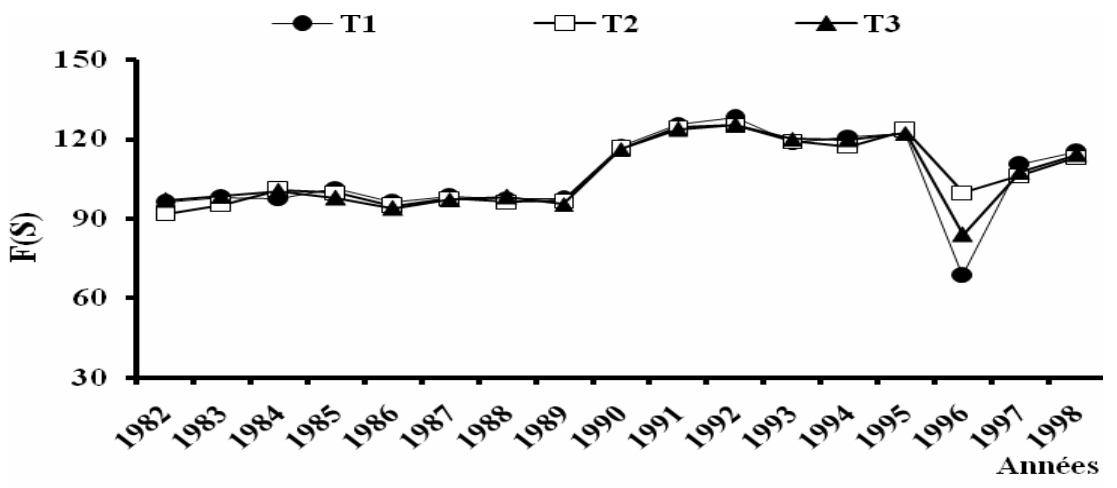

d) Nutrition en soufre

Figure 1 : Evolution des fonctions de production des cotonniers en azote (a), phosphore (b), potassium (c) et soufre (d) selon les modes de gestion des résidus de récolte de 1982 à 1998. $\mathrm{T} 1$ = gestion extensive des résidus + Fumure minérale $-\mathrm{T} 2$ = gestion semi-intensive des résidus + Fumure minérale + compost $-\mathrm{T} 3=$ gestion intensive des résidus + Fumure minérale + fumier.

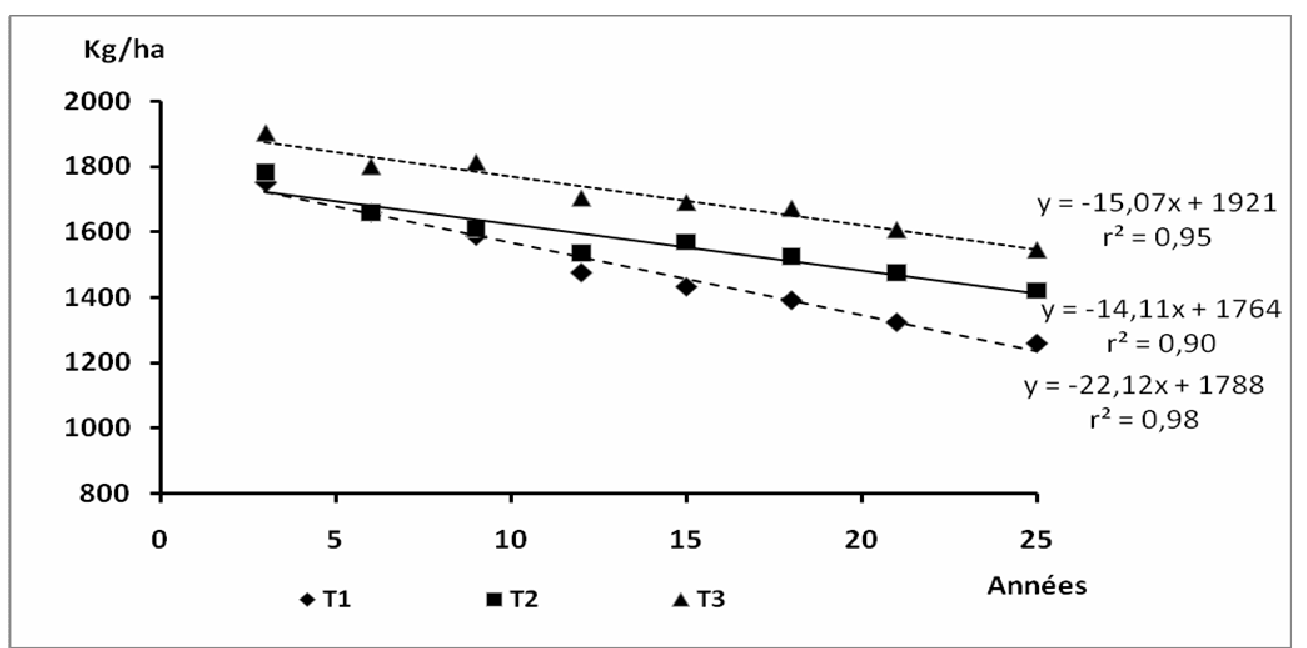

Figure 2 : Evolution des rendements du coton en fonction de la durée de mise en culture des terres et des modes de gestion des résidus de récolte.

$\mathrm{T} 1$ = gestion extensive des résidus + Fumure minérale $-\mathrm{T} 2=$ gestion semi-intensive des résidus + Fumure minérale + compost $-\mathrm{T} 3$ = gestion intensive des résidus + Fumure minérale + fumier. 


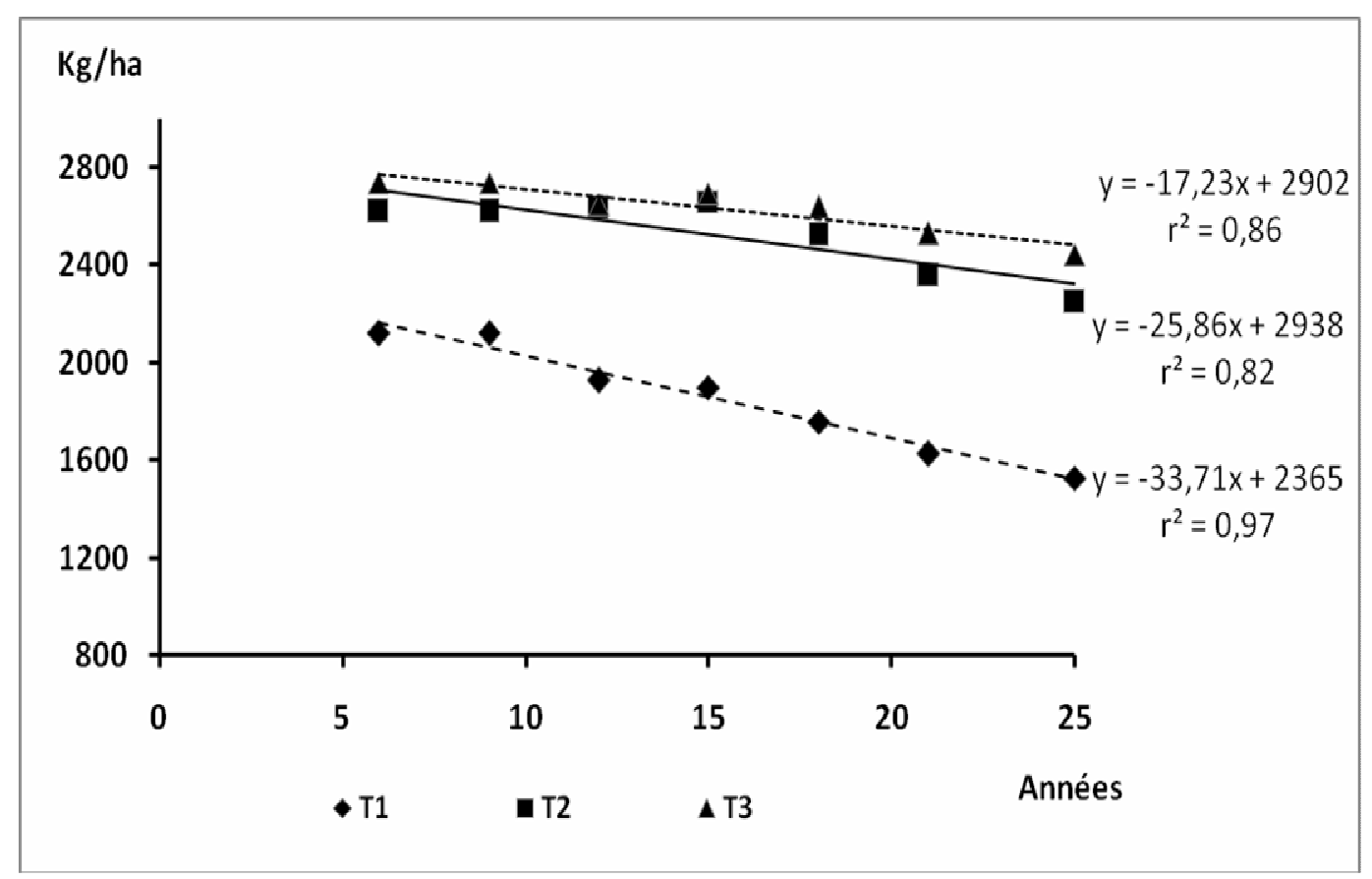

Figure 3 : Evolution des rendements du maïs en fonction de la durée de mise en culture des terres et des modes de gestion des résidus de récolte.

$\mathrm{T} 1=$ gestion extensive des résidus + Fumure minérale $-\mathrm{T} 2=$ gestion semi-intensive des résidus + Fumure minérale + compost $-\mathrm{T} 3=$ gestion intensive des résidus + Fumure minérale + fumier.

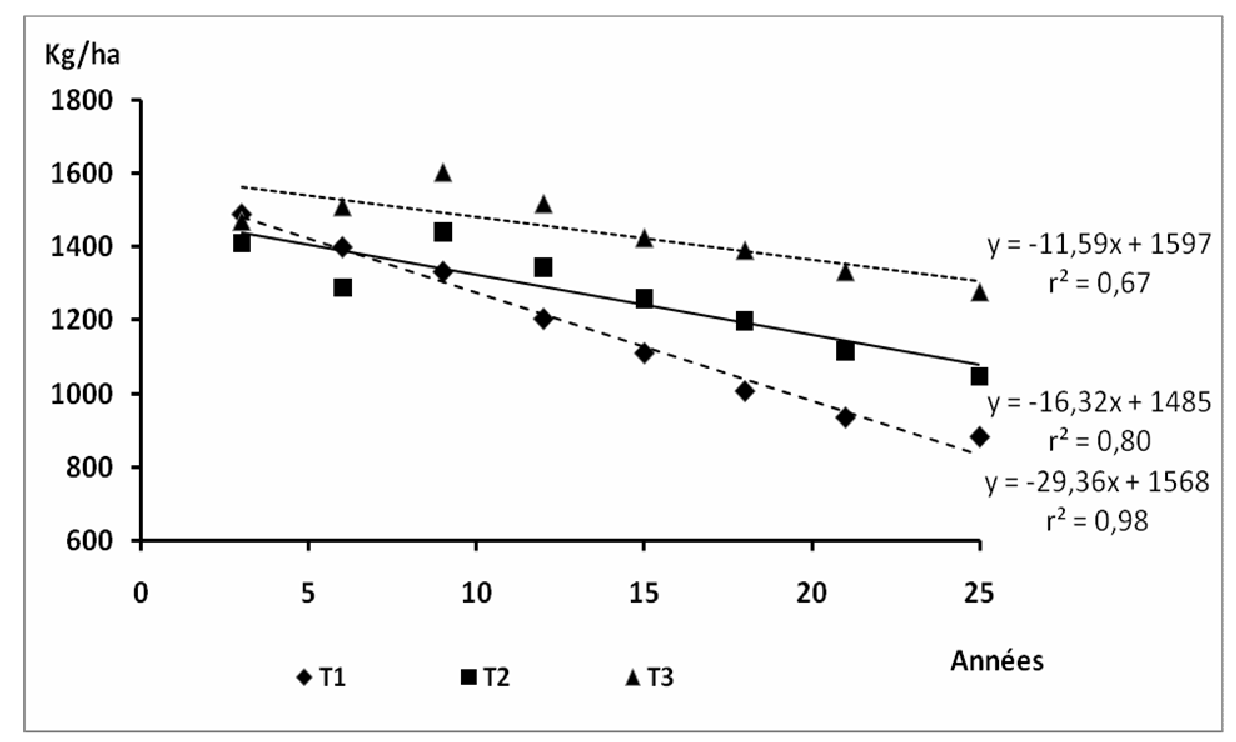

Figure 4 : Evolution des rendements du sorgho en fonction de la durée de mise en culture des terres et des modes de gestion des résidus de récolte.

$\mathrm{T} 1=$ gestion extensive des résidus + Fumure minérale $-\mathrm{T} 2=$ gestion semi-intensive des résidus + Fumure minérale + compost $-\mathrm{T} 3=$ gestion intensive des résidus + Fumure minérale + fumier. 
B. KOULIBALY et al. /Int. J. Biol. Chem. Sci. 4(6): 2120-2132, 2010

Tableau 1 : Pluviométrie et nombre de jours de pluie par an à Boni de 1982 à 2006.

\begin{tabular}{lcccccccc}
\hline & \multicolumn{9}{c}{ Pluviométrie mensuelle $(\mathbf{m m})$} & & $\begin{array}{c}\text { Cumul } \\
\text { Années }\end{array}$ & Mai & Juin & Juillet & Août & Septembre & Octobre & $\begin{array}{c}\text { Jours de } \\
\text { pluie/an }\end{array}$ \\
\cline { 2 - 6 } 1982 & 125 & 109 & 153 & 271 & 91 & 59 & 868 & 62 \\
1983 & 66 & 103 & 194 & 115 & 162 & 60 & 728 & 52 \\
1984 & 132 & 77 & 104 & 123 & 314 & 62 & 835 & 67 \\
1985 & 123 & 132 & 445 & 214 & 139 & 22 & 1098 & 53 \\
1986 & 125 & 105 & 242 & 209 & 255 & 21 & 988 & 66 \\
1987 & 57 & 153 & 232 & 243 & 132 & 17 & 840 & 49 \\
1988 & 27 & 153 & 250 & 352 & 280 & 53 & 1204 & 58 \\
1989 & 59 & 107 & 215 & 320 & 197 & 50 & 1057 & 67 \\
1990 & 51 & 106 & 154 & 177 & 176 & 77 & 841 & 57 \\
1991 & 161 & 142 & 140 & 175 & 87 & 76 & 855 & 61 \\
1992 & 66 & 142 & 201 & 159 & 208 & 38 & 823 & 55 \\
1993 & 19 & 67 & 169 & 351 & 154 & 62 & 831 & 50 \\
1994 & 82 & 201 & 285 & 253 & 193 & 108 & 1209 & 71 \\
1995 & 189 & 215 & 127 & 234 & 137 & 22 & 1006 & 63 \\
1996 & 115 & 113 & 187 & 346 & 205 & 77 & 1102 & 63 \\
1997 & 27 & 169 & 187 & 188 & 220 & 182 & 1015 & 68 \\
1998 & 188 & 110 & 186 & 249 & 209 & 14 & 970 & 59 \\
1999 & 112 & 57 & 251 & 425 & 232 & 64 & 1157 & 75 \\
2000 & 67 & 117 & 122 & 218 & 193 & 64 & 837 & 54 \\
2001 & 134 & 285 & 345 & 261 & 282 & 33 & 1353 & 58 \\
2002 & 38 & 143 & 109 & 245 & 154 & 69 & 758 & 42 \\
2003 & 73 & 150 & 147 & 234 & 241 & 22 & 924 & 59 \\
& & & & & & & &
\end{tabular}


B. KOULIBALY et al. / Int. J. Biol. Chem. Sci. 4(6): 2120-2132, 2010

\begin{tabular}{lcccccccc}
2004 & 110 & 85 & 283 & 176 & 92 & 16 & 781 & 46 \\
2005 & 79 & 101 & 123 & 150 & 240 & 47 & 840 & 40 \\
2006 & 58 & 143 & 131 & 267 & 282 & 107 & 987 & 57 \\
Maximum & 189 & 285 & 445 & 425 & 314 & 182 & 1353 & 75 \\
Minimum & 19 & 57 & 104 & 115 & 87 & 14 & 728 & 40 \\
Moyenne & $91 \pm 48$ & $131 \pm 49$ & $199 \pm 80$ & $238 \pm 76$ & $195 \pm 63$ & $57 \pm 37$ & $956 \pm 162$ & $58 \pm 9$ \\
\hline
\end{tabular}

Tableau 2 : Teneurs en éléments minéraux des feuilles de cotonniers prélevées à 70 jours après les semis.

\begin{tabular}{|c|c|c|c|c|c|c|c|c|c|c|}
\hline & Traitements & $\mathbf{N}$ & $\mathbf{P}$ & $\mathbf{K}$ & $\mathbf{S}$ & $\mathbf{C a}$ & Mg & $\mathbf{N a}$ & Cl & B \\
\hline & & & & & & $\%$ & & & & $\mathrm{mg} \mathrm{kg}^{-1}$ \\
\hline \multirow{3}{*}{$\begin{array}{l}\text { Cycle1 } \\
(1982-1984)\end{array}$} & $\mathrm{T} 1$ & $4,94 \pm 0,34$ & $0,32 \pm 0,02$ & $4,29 \pm 0,45$ & $0,41 \pm 0,10$ & $1,24 \pm 0,19$ & $0,42 \pm 0,06$ & $0,15 \pm 0,09$ & $1,77 \pm 0,16$ & $32,17 \pm 9,11$ \\
\hline & $\mathrm{T} 2$ & $4,86 \pm 0,35$ & $0,35 \pm 0,03$ & $4,57 \pm 0,56$ & $0,37 \pm 0,13$ & $1,15 \pm 0,12$ & $0,31 \pm 0,09$ & $0,13 \pm 0,09$ & $1,98 \pm 0,17$ & $39,33 \pm 14,15$ \\
\hline & $\mathrm{T} 3$ & $4,70 \pm 0,60$ & $0,31 \pm 0,05$ & $4,37 \pm 0,87$ & $0,45 \pm 0,08$ & $1,20 \pm 0,17$ & $0,34 \pm 0,07$ & $0,14 \pm 0,11$ & $1,57 \pm 0,26$ & $31,67 \pm 10,97$ \\
\hline \multirow{3}{*}{$\begin{array}{l}\text { Cycle } 2 \\
(1985-1987)\end{array}$} & $\mathrm{T} 1$ & $4,22 \pm 0,34$ & $0,28 \pm 0,03$ & $3,30 \pm 0,73$ & $0,53 \pm 0,08$ & $1,26 \pm 0,09$ & $0,47 \pm 0,09$ & $0,06 \pm 0,03$ & $0,92 \pm 0,13$ & $32,83 \pm 3,13$ \\
\hline & $\mathrm{T} 2$ & $4,17 \pm 0,37$ & $0,27 \pm 0,04$ & $3,31 \pm 0,49$ & $0,50 \pm 0,06$ & $1,16 \pm 0,04$ & $0,40 \pm 0,02$ & $0,04 \pm 0,01$ & $0,82 \pm 0,11$ & $33,33 \pm 4,62$ \\
\hline & $\mathrm{T} 3$ & $4,08 \pm 0,62$ & $0,28 \pm 0,05$ & $3,59 \pm 0,67$ & $0,45 \pm 0,09$ & $1,19 \pm 0,05$ & $0,38 \pm 0,02$ & $0,04 \pm 0,02$ & $0,77 \pm 0,23$ & $35,00 \pm 1,00$ \\
\hline \multirow{3}{*}{$\begin{array}{l}\text { Cycle } 3 \\
(1988-1990)\end{array}$} & $\mathrm{T} 1$ & $4,63 \pm 0,49$ & $0,32 \pm 0,07$ & $4,50 \pm 0,85$ & $0,69 \pm 0,15$ & $1,72 \pm 0,15$ & $0,46 \pm 0,08$ & $0,04 \pm 0,02$ & $1,04 \pm 0,25$ & $30,54 \pm 8,94$ \\
\hline & $\mathrm{T} 2$ & $4,70 \pm 0,73$ & $0,33 \pm 0,07$ & $4,27 \pm 0,28$ & $0,60 \pm 0,16$ & $1,65 \pm 0,32$ & $0,46 \pm 0,15$ & $0,04 \pm 0,02$ & $0,81 \pm 0,24$ & $31,51 \pm 9,96$ \\
\hline & $\mathrm{T} 3$ & $4,83 \pm 0,50$ & $0,32 \pm 0,06$ & $3,96 \pm 0,41$ & $0,64 \pm 0,14$ & $1,72 \pm 0,13$ & $0,53 \pm 0,03$ & $0,05 \pm 0,02$ & $0,73 \pm 0,09$ & $30,96 \pm 10,45$ \\
\hline \multirow{2}{*}{$\begin{array}{l}\text { Cycle } 4 \\
(1991-1993)\end{array}$} & $\mathrm{T} 1$ & $4,29 \pm 0,36$ & $0,45 \pm 0,15$ & $3,88 \pm 0,31$ & $0,89 \pm 0,39$ & $1,76 \pm 0,06$ & $0,59 \pm 0,06$ & $0,06 \pm 0,02$ & $1,06 \pm 0,35$ & $32,67 \pm 4,45$ \\
\hline & $\mathrm{T} 2$ & $4,32 \pm 0,33$ & $0,45 \pm 0,14$ & $3,91 \pm 0,37$ & $0,89 \pm 0,61$ & $1,61 \pm 0,08$ & $0,51 \pm 0,12$ & $0,04 \pm 0,02$ & $0,97 \pm 0,41$ & $34,83 \pm 9,19$ \\
\hline
\end{tabular}


B. KOULIBALY et al. / Int. J. Biol. Chem. Sci. 4(6): 2120-2132, 2010

\begin{tabular}{|c|c|c|c|c|c|c|c|c|c|c|}
\hline & $\mathrm{T} 3$ & $4,56 \pm 0,46$ & $0,43 \pm 0,13$ & $3,77 \pm 0,32$ & $0,88 \pm 0,52$ & $1,68 \pm 0,05$ & $0,54 \pm 0,06$ & $0,04 \pm 0,03$ & $0,87 \pm 0,35$ & $32,33 \pm 0,94$ \\
\hline \multirow{3}{*}{$\begin{array}{l}\text { Cycle } 5 \\
(1994-1996)\end{array}$} & $\mathrm{T} 1$ & $1,91 \pm 0,50$ & $0,59 \pm 0,05$ & $4,41 \pm 0,91$ & $1,00 \pm 0,41$ & $2,34 \pm 0,29$ & $0,52 \pm 0,16$ & $0,29 \pm 0,11$ & $0,93 \pm 0,20$ & - \\
\hline & $\mathrm{T} 2$ & $2,03 \pm 0,55$ & $0,61 \pm 0,07$ & $4,42 \pm 0,33$ & $0,94 \pm 0,37$ & $2,52 \pm 0,41$ & $0,43 \pm 0,14$ & $0,52 \pm 0,27$ & $0,94 \pm 0,15$ & - \\
\hline & $\mathrm{T} 3$ & $2,00 \pm 0,56$ & $0,58 \pm 0,05$ & $4,68 \pm 1,30$ & $0,81 \pm 0,33$ & $2,26 \pm 0,08$ & $0,66 \pm 0,24$ & $0,44 \pm 0,19$ & $1,45 \pm 0,35$ & - \\
\hline \multirow{3}{*}{$\begin{array}{l}\text { Cycle } 6 \\
(1997-1998)\end{array}$} & $\mathrm{T} 1$ & $3,70 \pm 0,09$ & $0,54 \pm 0,06$ & $4,54 \pm 0,18$ & $0,46 \pm 0,04$ & - & - & - & - & - \\
\hline & $\mathrm{T} 2$ & $3,92 \pm 0,14$ & $0,65 \pm 0,09$ & $4,25 \pm 0,75$ & $0,50 \pm 0,10$ & - & - & - & - & - \\
\hline & $\mathrm{T} 3$ & $3,87 \pm 0,10$ & $0,61 \pm 0,08$ & $3,72 \pm 0,61$ & $0,47 \pm 0,06$ & - & - & - & - & - \\
\hline \multicolumn{11}{|l|}{ Analyses } \\
\hline \multicolumn{2}{|l|}{ Cycles } & $<0,0001(\mathrm{~s})$ & $<0,0001(\mathrm{~s})$ & $0,011(\mathrm{~s})$ & $0,004(\mathrm{~s})$ & $<0,0001(\mathrm{~s})$ & $0,001(\mathrm{~s})$ & $0,008(\mathrm{~s})$ & $<0,0001(\mathrm{~s})$ & $0,341(\mathrm{~ns})$ \\
\hline \multicolumn{2}{|l|}{ Traitements } & $0,771(\mathrm{~ns})$ & 0,289 (ns) & $0,644(\mathrm{~ns})$ & $0,685(\mathrm{~ns})$ & $0,580(\mathrm{~ns})$ & 0,195 (ns) & 0,728 (ns) & 0,098 (ns) & $0,382(\mathrm{~ns})$ \\
\hline \multicolumn{2}{|c|}{ Cycles $\mathrm{x}$ traitements } & $<0,0001(\mathrm{~s})$ & $0,004(\mathrm{~s})$ & 0,177 (ns) & $0,027(\mathrm{~s})$ & $<0,0001(\mathrm{~s})$ & $0,011(\mathrm{~s})$ & $0,001(\mathrm{~s})$ & $<0,0001(\mathrm{~s})$ & $0,216(\mathrm{~ns})$ \\
\hline
\end{tabular}

$\mathrm{N}=$ teneur en azote $-\mathrm{P}=$ teneur en phosphore $-\mathrm{K}=$ teneur en potassium $-\mathrm{S}=$ teneur en soufre $-\mathrm{Ca}=$ teneur en calcium $-\mathrm{Mg}=$ teneur en magnésium $-\mathrm{Na}=$ teneur en sodium $-\mathrm{Cl}=$ teneur en chlore (exprimées en $\%$ de matière sèche) $\mathrm{B}=$ teneur en bore (exprimée en $\mathrm{mg} \mathrm{kg}^{-1}$ de matière sèche).

Les valeurs précédées du signe \pm représentent les écart-types.

- : donnée non disponible

$\mathrm{s}$ : significatif - ns : non significatif, au seuil de signification de 5\% selon le test de Fisher. 


\section{DISCUSSION}

Les analyses foliaires permettent de prévoir l'apparition probable d'une déficience minérale et de prendre des mesures de fertilisation nécessaires pour l'éviter (Hassan et Arshad, 2008). La satisfaction des besoins des cotonniers pour la plupart des éléments minéraux montre que la fumure minérale (T1) présente sur ce sol, une efficacité prolongée avec l'adjonction du phosphate naturel. Les premières déficiences nutritionnelles en azote et en potassium ne sont apparues sur les cotonniers qu'au bout de 13 années d'étude. Ce résultat confirme les travaux de Braud (1987) qui a observé avec la fertilisation minérale, une nutrition correcte des cotonniers pendant plus de 10 ans. Le recyclage des résidus en compost ou fumier n'a pas d'effets significatifs sur les teneurs en éléments minéraux des cotonniers qui par contre présentent des différences significatives entre les cycles de rotation qui seraient liées probablement à l'effet de la pluviosité très déterminante sur la mobilisation et l'absorption des éléments. La nutrition azotée du cotonnier est liée à la durée utile de la saison des pluies et à l'intensité de la pluviosité qui augmente sensiblement la lixiviation (Crozier et al., 2004 ; Girma et al., 2007). De tels facteurs pourraient expliquer la déficience azotée. La pluviosité relativement élevée de 1994 à 1996 qui devrait favoriser l'absorption du potassium a, au contraire, induit une nutrition potassique déficiente à cause probablement d'une faible activité du système racinaire ou d'une faible disponibilité de cet élément dans le sol (Dakouo, 1994 ; Bednarz et Oosterhuis, 1999). La nutrition phosphatée des cotonniers a été correcte ce qui suggère de bonnes conditions de dissolution $\mathrm{du}$ phosphate naturel en application directe ou incorporé au compost (FAO, 2004; Lompo et al., 2009). La déficience exceptionnelle en soufre sur le témoin sans restitution organique semble liée au déficit d'azote du fait des rapports étroits entre ces deux éléments. En 17 années d'observation, les fumures associées aux trois modes de gestion des résidus de récolte se sont révélées efficaces et adaptées pour les besoins $\mathrm{du}$ cotonnier en calcium, en magnésium et surtout en bore dont les teneurs dépassent largement le niveau critique de 21 $\mathrm{mg} \mathrm{kg}^{-1}$ (Braud, 1987).

La baisse des rendements des cultures avec la durée d'exploitation des terres est liée à une dégradation du sol mise en évidence par les analyses de sol qui ont montré une désaturation progressive du complexe (en calcium et magnésium) et une diminution de la matière organique accentuée par l'exportation des résidus de récolte (Koulibaly et al., 2010). Le recyclage des résidus en compost ou fumier est nécessaire voire indispensable, puisque Ouattara et al. (2006), rapportent que la fumure minérale seule ne permet de maintenir les rendements des cultures que pendant seulement 5 à 6 années, ce qui met en péril la durabilité du système de culture. La baisse générale du potentiel de production est aussi imputable à la persistance des déficits pluviométriques qui caractérisent les variations du climat au Burkina Faso (Bénoît, 2008; Barro et al., 2009). Selon Abou Abba et al. (2006), les conséquences des déficits pluviométriques sur les productions agricoles sont d'autant plus importantes que le système d'exploitation est extensif, d'où la nécessité d'une gestion rationnelle des résidus de récolte. Les restitutions organiques permettent selon Vullioud et al. (2004) d'améliorer les propriétés bio-physicochimiques du sol, surtout sa disponibilité en éléments nutritifs. Bien qu'aucune amélioration nette de la nutrition minérale des cotonniers n'ait été observée avec le recyclage des résidus en compost (T2) ou en fumier (T3), ces deux modes de gestion des résidus permettent de réduire au minimum la baisse des rendements avec la durée d'exploitation des terres.

\section{Conclusion}

La fumure minérale seule (gestion extensive des résidus de récolte) ou associée au compost (gestion semi-intensive) ou au 
fumier (gestion intensive des résidus culturaux), permet une nutrition minérale globale correcte des cotonniers pendant au moins 13 ans avant l'apparition des premières déficiences minérales. La nutrition phosphatée correcte des cotonniers met en évidence l'efficacité et l'intérêt du phosphate naturel dont la valorisation devrait permettre de réduire le coût de la fertilisation dans les systèmes de culture coton-céréales. A défaut de maintenir le potentiel de production, l'enfouissement au sol des résidus et leur recyclage en fumure organique, combinés à la fumure minérale, réduisent la baisse des rendements liée à l'exploitation continue des terres. L'étude montre l'intérêt d'une gestion intégrée des résidus de récolte combinée à l'utilisation du phosphate naturel pour la productivité des systèmes de culture cotoncéréales en zone cotonnière.

\section{REMERCIEMENTS}

Les auteurs remercient la Société Burkinabè des Fibres Textiles (SOFITEX) pour son appui financier et matériel dans la réalisation de cette étude. Ils remercient vivement tous les chercheurs et techniciens du Programme Coton de l'Institut de l'Environnement et de Recherches Agricoles (INERA) pour leur contribution à l'étude depuis sa mise en place en 1982.

\section{REFERENCES}

Abou Abba A, Hofs JL, Mergeai G. 2006. Relever les défis environnementaux pour les filières cotonnières d'Afrique de l'Ouest et du Centre. Biotechnol. Agron. Soc. Environ., 10(4): 351-359.

Agoumé V, Birang AM. 2009. Impact of landuse systems on some physical and chemical soil properties of an oxisol in the humid forest zone of southern Cameroon. Tropicultura, 27(1): 15-20.

Barro A, Zougmoré R, Sédogo MP. 2009. Evaluation de la faisabilité de trois types de travail du sol : application du modèle Sarra dans le Plateau central au Burkina Faso. Sécheresse, 20(4): 338-345.
Bednarz CW, Oosterhuis DM. 1999. Physiology of the onset of potassium deficiency in cotton. Journal of Plant Nutrition, 22: 303-313.

Bénoît E. 2008. Les changements climatiques: vulnérabilité, impacts et adaptation dans le monde de la médecine traditionnelle au Burkina Faso. Vertigo - La Revue en Sciences de l'Environnement, 8(1): 1-12.

Berger M. 1996. L'amélioration de la fumure organique en Afrique Soudanosahélienne. Agric. Dév., hors série, fiches techniques. CIRAD éditions : Montpellier ; 29.

Braud M. 1974. Le contrôle de la nutrition minérale du cotonnier par analyses foliaires. Cot. Fib. Trop., 29(2): 215-225.

Braud M. 1987. La fertilisation d'un système de culture dans les zones cotonnières soudanosahéliennes. Cot. Fib. Trop., 8: 35.

Bunasols. 1987. Méthodes d'analyse physique et chimique des eaux et du sol. Document technique $\mathrm{n}^{\circ} 3$, Ouagadougou, Burkina Faso, p. 159.

Crozier CR, Walls B, Hardy DH, Barnes JS. 2004. Response of cotton to P and K soil fertility gradients in North Carolina. Journal of Cotton Science, 8(3): 130-141.

Dakouo D. 1994. Les carences en potassium sur cotonnier (Gossypium hirsutum L.) dans les systèmes de culture: cas de la zone cotonnière ouest du Burkina. Thèse docteur-Ingénieur; Option sciences Agronomiques, Université Nationale de Côte d'Ivoire, Abidjan, p. 141.

FAO. 2004. Utilisation des phosphates naturels pour une agriculture durable. Bulletin Fao, Engrais et nutrition végétale, 13, p.144.

Girma K, Teal RK, Freeman KW, Boman RK, Raun WR. 2007. Cotton lint yield and quality as affected by applications of $\mathrm{N}$, $\mathrm{P}$, and $\mathrm{K}$ fertilizers. Journal of Cotton Science, 11(1): 12-19.

Hassan ZU, Arshad M. 2008. Evaluating factors affecting cotton tolerance to potassium deficiency stress using path 
analysis. Int. J. Agri. Biol., 10(5): 511516.

Koulibaly B, Traoré O, Dakuo D, Zombré PN, Bondé D. 2010. Effets de la gestion des résidus de récolte sur les rendements et les bilans culturaux d'une rotation cotonnier-maïs-sorgho au Burkina Faso. Tropicultura, 28(3): 184-189.

Lompo F, Segda Z, Gnankambary Z, Ouandaogo N. 2009. Influence des phosphates naturels sur la qualité et la biodégradation d'un compost de pailles de maïs. Tropicultura, 27(2): 105-109.

Olina Bassala JP, M'Biandoun M, Ekorong JA, Asfom P. 2008. Evolution de la fertilité des sols dans un système cotonnier céréales au Nord Cameroun: diagnostic et perspectives. Tropicultura, 26(4): 240-245.

Ouattara B, Ouattara K, Serpentié G, Mando A, Sédogo MP, Bationo A. 2006. Intensity cultivation induced effects on soil organic carbon dynamic in the western cotton area of Burkina Faso. Nutr. Cycl. Agroecosyst., 76: 331-339.
Ouédraogo E, Mando A, Stroosnijder L. 2006. Effects of tillage, organic ressources and nitrogen fertiliser on soil carbon dynamics and crop nitrogen uptake in semi-arid West Africa. Soil Till. Res., 91(1-2): 57-67.

Reiter MS, Reeves DW, Burmester CH. 2008. Cotton nitrogen management in a highresidue conservation system: source, rate, method, and timing. Soil Sci. Soc. Am. J., 72: 1330-1336.

Traoré O, Somé NA, Traoré K, Somda K. 2007. Effect of land use change on some important soil properties in cotton-based farming system in Burkina Faso. Int. J. Biol. Chem. Sci., 1(1): 7-14.

Vanlauwe B, Diels J, Sanginga N, Merckx R. 2005. Long-term integrated soil fertility management in South-western Nigeria: crop performance and impact on the soil fertility status. Plant and Soil, 273(1-2): 337-354.

Vullioud P, Mercier E, Ryser JP. 2004. Bilan de 40 ans d'essai portant sur différentes fumures organiques (Changrin 19632003). Revue Suisse d'Agriculture, 36(2): 43-51. 